\title{
Electrodeposition route to synthesize cigs films - an economical way to harness solar energy
}

\author{
N.G. Renganathan and M. Venkata Subramanian and S. Mohan* \\ Vel Tech Dr. RR \& Dr. SR Technical University,Chennai, 600 062, INDIA. \\ *EMFT Division, Central Electrochemical Research Institute, Karaikudi,630006, INDIA \\ "Corresponding Author: e-mail: N.G. Renganathan (ngrenganthan@gmail.com)
}

\begin{abstract}
Copper Indium Gallium Selenide has become one of the most highly promising absorber materials for thin film solar cells due to its exceptional semiconductor characteristics. Various attempts have been made in the recent years to scale up the production of these films. In this review the difficulty in scaling up of the existing technologies, the necessity of nano-particles of CIGS for solar cells, how the charge separation in this nano scale photovoltaic (PV) materials occurs which help in absorption of radiation, and the electro-deposition route, a low cost one, produces thin film solar cells are analyzed. The envisaged pulse electro-deposition route will not only produce the required nano-scale CIGS films but also a route to large scale economical production of thin films solar cells.
\end{abstract}

Keywords: Solar cell; Thin film; CuInGaSe ; Electro-deposition

\section{Introduction}

Copper Indium Gallium Selenide has become one of the most highly promising absorber materials for thin film solar cells due to its exceptional semiconductor characteristics. Various attempts have been made in the recent years to scale up the production of these films. Most of the labs developing CIGS films have used physical evaporation (Gur et al., 2005; Wu et al., 2008; Kavcar et al., 1992; Panthani et al., 2008), sputtering techniques, spin coating, selenization of Ga-rich electrodeposited precursors (Kang et al., 2009) and mist deposition. In all these attempts the aim was to synthesize the film and characterize for the application of solar cells. Electro-deposition is the route by which minimum particle size and large scale scaling up can be envisaged. In this review, attempts were made to address the following points and answers in brief: (i) The difficulty in scaling up of the existing technologies; (ii) Why nano-particles of CIGS are needed for solar cells; (iii) How the charge separation in this nanoscale photovoltaic (PV) materials occurs which help in absorption of radiation; (iv) The electro-deposition route produces thin film solar cells; (v) The envisaged pulse electro-deposition route will not only produce the required nano-scale CIGS films but the prospects of scaling up this technique to large scale economical production of thin film solar cells

\section{The difficulty in scaling up of the existing technologies}

The main reason is that copper Indium selenide or copper indium gallium selenide is a multi component compound with a complex phase diagram. It is believed to have more than twenty phases. It is also an efficient self doping semiconductor. This is because of low formation energy of point defects and their complexes. For an efficient solar cell good crystal quality with a large micrometer grain size is desirable. During the film formation process complex reaction processes involving elemental, binary, ternary and quaternary intermediate compounds occur depending on the different film growth methods. It is also reported that during the second stage of the co-evaporation process the (In, Ga) $)_{2} \mathrm{Se}_{3}$ layer is converted to $\alpha$-CIGS via gamma -CIGS and beta CIGS. In these co-evaporation processes it is not able to control the particle size which is vital for absorption property. In the formation of p-type CIGS film formation, especially during physical vapor deposition or co-evaporation processes, the diffusion of the constituent elements has to take place in the solid/liquid reactions between the intermediate phases. Phase segregation and 
phase transformations also complicate the formation CIGS films. Unlike binary conductors CIGS film preparation needs highly controllable technology. This is essential with respect to materials supply during deposition and phase formation processes. Hence chemical reaction process needs to be fully understood before scaling up operation. Another main reason in the scaling up of the film is cost. The production cost of cadmium telluride thin film solar cells is $85 \mathrm{c}$ per peak watt (Wp). But the production cost of CIGS thin film solar cell is $\$ 1.5-1.75 / \mathrm{Wp}$ (Dhere, 2011). The production cost of CIGS PV modules lags considerably behind that of CdTe PV modules. This is because there are several scale-up issues as already addressed. The various technologies for the production of CIGS films are: (1) Co-evaporation; (2) Reactive sputtering of $\mathrm{Cu}, \mathrm{In}, \mathrm{Ga}$ with selenium; (3) Selenization /or sulfurization of sputter deposited $\mathrm{Cu}-\mathrm{In}$-Ga precursor; (4) Selenization or sulfurization of $\mathrm{Cu}-\mathrm{In}$-Ga precursor deposited by nonvacuum techniques; (5) Electro-deposition; and (6) Doctor blade.

Large volume production of selenization or sulfurization of magnetron sputtered $\mathrm{Cu}-\mathrm{In}$-Ga precursors have been attempted by certain companies like Showa Shell, Honda, Sulfurcell and Avancis, which is another issue in the scaling up process. Showa Shell has achieved $\sim 15 \%$ efficiency on a limited area and $\sim 13 \%$ on a bigger area of $\sim 7000 \mathrm{~cm}^{2}$ PV modules by selenization or sulfurization of magnetron $\mathrm{Cu}$-In-Ga precursors. However, these processes are prone to toxicity. To overcome this, metal organic selenides are preferred. Yet another issue in the scaling up is large quantities of materials are needed in the non-vacuum processes. In these processes, based on thickness of the films, two technologies can be thought of. One is thin film technology wherein thin films are grown atom by atom, or molecule by molecule as in vacuum evaporation, sputtering, chemical bath deposition, chemical vapor deposition, electro-deposition etc. On the other hand thick films are grown by agglomeration of particles from slurry or suspensions e.g. screen printing, ink jet printing or doctor blade technique. Ink jet and doctor blade techniques use the nanoparticle of the precursor films onto the substrate which will be processed further for sulfurization or selenization. In these processes there are engineering challenges for coating on a larger surface and for further processing the films like sulfurization or selenization. These methods may not be cost effective. This argument also holds well in forming a hetero-junction partner $\mathrm{CdS}$ layer.

In a nut shell, the aim of large scale production of CIGS film depends on volume, speed and yield of the product. The basic R\& $\mathrm{D}$ depends on concentrating the optimization of the operational windows of various processing techniques to consistently achieve high efficiency of $\sim 20 \%$ in small area $\sim 0.4 \mathrm{~cm}^{2}$. Then the laboratory process is transferred to small scale industry to scale up to $50-100 \mathrm{~cm}^{2}$. The experience is then transferred to pilot plant level $0.5-5 \mathrm{MW} /$ year. To produce, larger area CIGS cells of $0.1-1 \mathrm{~m}^{2}$ ( $>20 \mathrm{MW} /$ year), cost effective PV circuits and modules have to be developed. All thin film technologies have a high cost reduction potential at high production volumes. In future, combination of high production volumes with high throughput, sufficient yield and superior quality will be a challenging one. In this aspect ZSW, a company from Germany with Wurth group engaged to commercialize CIGS technology (Powalla and Dimmler, 2001). A maximum efficiency of $10 \%$ is reported for the CIGS films grown by sequential sputtering, electro-deposition and physical vapor deposition. These cells are fabricated using CdS heterojunction partners. The efficiency from these cells depends on the microstructure of the sputtered films which depend upon annealing conditions. It is also noted that higher efficiencies can be obtained if the films have deficiencies in copper where concentration of holes will be predominant. Impurity phases like $\mathrm{Cu}_{1.8} \mathrm{Se}$ should be avoided to have more efficient solar cells. Bulk conductivity of the film should preferably be p-type whereas the surface conductivity should be n-type (Hermann et al., 2001).

Companies such as Wurth Solar, ZSW, Gloabal Solar, Sulfurcell, Shell Solar Industries and Showa Shell Seiku are involved in manufacturing of PV modules using CIGS technology. Of the various technologies for the fabrication of CIGS absorber, co evaporation of $\mathrm{Cu}, \mathrm{In}, \mathrm{Ga}$, and $\mathrm{Se}$ is used by Wurth Solar and Global Solar. The co-evaporation technique is not suitable to coat large areas with sufficient process stability and homogeneity. Wurth solar involves in fabricating monolithically interconnected CIGS solar cells on a larger area and the efficiency achieved is also to the extent of $13 \%$. Their current production is $3 \mathrm{MW} / \mathrm{year}$. Global Solar adopts Co-evaporation and the method adopted is roll-to -roll coating of CIGS absorber. The efficiency achieved is $10.4 \%$ and this company is also able to coat the film over large area and it has the capacity of the plant of 3 MW/year. Shell and Saint Gobain industries believe that non silicon based solar technologies such as CIS are well placed to potentially become competitive with conventional sources of electricity.

Showa shell is expecting to increase the capacity to $20 \mathrm{MW} / \mathrm{year}$ in the years to come. In comparing the PV manufacturing technologies it is worth mentioning that whatever the technology adopted for CdTe it can be easily adopted for CIGS. Solar has perfected the manufacturing of CdTe solar cell using vapor transport deposition of CdS and CdTe and the efficiency was $10.2 \%$ for a reasonable aperture area. In CIGS, PV module production, sputter deposition of $\mathrm{Mo}, \mathrm{ZnO}$ and $\mathrm{ZnO}: \mathrm{Al}$ and laser and mechanical scribing are easily adoptable to in line production. The process of Selenization/sulfurization is a batch process. Chemical Bath Deposition (CBD) of CdS deposition is a batch process. If a dry process like sputtering could be developed for the deposition of a hetero-junction partner layer such as $\mathrm{CdS}$ or $\mathrm{ZnO}$ then the manufacture could be easily adopted as in line process and manufacture of CIGS could be easily adopted. In principle the rate of deposition of all the layers should be matched perfectly in a roll to roll process. If there is a faster process then the roll-to-roll process has to be modified to suit the faster process. For monolithic interconnection polymer sheets may be used for high temperature deposition of CIGS layers. In many ways the nonvacuum process is similar to crystalline solar cells. In non-vacuum process the absorber must be synthesized in a non-vacuum environment.

Among the thin film technologies, the efficiencies of CIGS cell and module are the highest. But the CIGS cell fabrication technology is more complex. Worldwide PV cell and module annual production is estimated to be $1.75 \mathrm{GW} /$ year in the year 2006 
and is expected to grow 5, 34, and $380 \mathrm{GW} /$ year respectively in the year 2010, 2020 and 2030. Within the thin film PV, CIGS technology has the highest potential for improvement with efficiencies up to $25 \%$ for cells and $18 \%$ for modules.

Honda's thin film CIGS has a new production process. It has reached the highest level of PV transfer efficiency for a thin film cell and this is the equivalent to the conventional crystalline silicon solar cells. It has achievement of $50 \%$ reduction in the use of energy in the manufacturing process of a solar cell. CIGS films are prepared by selenization of CuInGa single-layer metallic precursor (Su et al., 2011). In this process CuInGa metallic precursors are deposited onto soda lime glass by Direct Current magnetron sputtering system using a CuInGa ternary alloy target with a precise composition of $\mathrm{Cu}: \mathrm{In}: \mathrm{Ga}$ of 1:0.7:0.3. The precursor films are then made to react with Se vapor in vacuum evaporation system. In spite of the complicated process, large facetted grains with a single chalcopyrite phase is reported with a preferred orientation along $(1,1,2)$ plane. Still the process will not be a viable one for large scale production of the film. Polycrystalline CIGS thin film absorbers were prepared by selenizing electrodeposited precursors (Kang et al., 2010). In this two different levels of selenium contents were prepared and the performance characteristics were compared. The results of XRF, XRD, SEM, and illuminated I-V indicate that the absorber layers processed from less amount of selenium shows better crystalline quality and increased gallium incorporation. The cell performance was also improved compared to selenium rich precursors. $\mathrm{CuInSe}$ (CIS), $\mathrm{Cu}$ (InGa) $\mathrm{Se}_{2}$ (CIGS) and $\mathrm{CuGaSe}_{2}$ (CGS) solar cells were fabricated on a flexible zirconia substrates (Ishizuka et al., 2010). Alkali doping was tried by using alkali silicate thin layers (ASTL) deposited on substrates prior to sputtering of the Mo back contact. By ASTL layer, it was demonstrated that cell efficiency was increased regardless of the In/Ga composition in CIGS. By alkali element incorporation in CIGS films it was shown that elemental inter diffusion of In and Ga during growth was reduced. This had resulted in a decrease of the nominal band gap energy of CIGS layer due to steep Ga composition gradient present in CIGS layer. $\mathrm{CuInSe}_{2}$ (CIS) and $\mathrm{Cu}(\mathrm{In}, \mathrm{Ga}) \mathrm{Se}_{2}$ (CIGS) films as solar cell absorbers have been fabricated from electro deposition method (Oda et al., 2010). In-Se/CIS and CIS/CuGaSe 2 bilayers, InSe /CIS bilayers were intermixed by annealing at $600^{\circ} \mathrm{C}$ for 10 minutes and the CIS films with large grains were obtained but the efficiency was only $2.2 \%$. A non-vacuum process for fabrication of CIGS absorber layer has been reported (Ahn et al., 2008). In this CIGS nano- particle precursors has been prepared by a low temperature colloidal route reacting the starting materials (CuI, $\mathrm{InI}, \mathrm{GaI}_{3}$ and $\mathrm{Na}_{2} \mathrm{Se}$ ) in organic solvents. The film is obtained by doctor blade method by casting nano-particle precursor material in a suitable organic solvent over Mo/glass substrate and this film is used as solar absorbing material.

\section{Why nano-particles of CIGS are essential for solar absorbers?}

One needs to span across both fundamental and scientific disciplines for addressing materials challenges for improving PV cells. For predicting and designing novel materials with desired behaviors one needs to understand the phenomena at a fundamental level. From the computational materials science it is learnt that novel nano-materials are amenable for charge separation in PV cells. As it is suggested earlier, charge separation (Kanai et al., 2010) of the exciton is induced by introducing another material, generally a dopant. In nano-materials the electronic structure can be tuned. This is usually done by quantum confinement and topological symmetries. It is also possible to control the intrinsic properties of a single nano-material to induce charge separation without doping material. The excitons in nano-crystal can be viewed as electrons trapped in a finite potential well. This leads to discrete allowed energy levels for electrons. These allowed energy levels produce band gaps where number of electrons is allowed. When photons are absorbed, number of electrons is excited from a low energy state to an unoccupied, high energy state by absorbing the exact amount of energy from the photon that matches the band gap. This band gap is highly dependent on the size of the nano crystal. Size dependent shift in the exciton energy with respect to the band gap can be given as: (Wang and Herron, 1991).

$\Delta \mathrm{E}=\frac{\mathrm{h}^{2} \pi^{2}}{2 \mathrm{R}^{2}\left(1 / \mathrm{m}_{\mathrm{e}}+1 / \mathrm{m}_{\mathrm{h}}\right)}-\frac{1.768 \mathrm{e}^{2}}{\varepsilon \mathrm{R}}-0.248 \mathrm{e}^{*} R_{\mathrm{y}}$

Where $R_{\mathrm{y}}$ is the effective Rydberg energy, $\mathrm{m}_{\mathrm{e}}$ and $\mathrm{m}_{\mathrm{h}}$ are effective mass of electron and holes respectively and $\varepsilon$ is the dielectric constant. Thus the band gap can be systematically tuned by changing nano crystal size.

\section{How the charge separation in these nano-scale photovoltaic materials occur which help in absorption of radiation:}

As reported earlier, supra molecular nano architectures for light energy conversion largely depend on the grain structures (Hasobe, 2010). The first and foremost criterion is size and morphological control of molecular assembly for efficient transport of charge carriers. To develop high efficiency photo- voltaics solar cells have to be designed from low dimensional semi conductor structures. For achieving long wavelength absorption in solar cells incorporation of Quantum Dots is necessary (Kamat, 2008). Moreover these solar cells are not limited by selection rules for optical transitions in Quantum Wells and their size helps to mitigate strain effects in the active layer. 
The first step in understanding is the absorption of solar photon by the active material. This will result in an electronic excitation in the material. This will better be achieved in the case of nano- materials. In the solar flux spectrum photon absorption is a quite infrequent event in the nano-scale. But we may describe the photo excitation process is primarily a single photon absorption process. In PV process the excited electron can be viewed as a distinguishable particle with an explicit position. Subsequent step can be described as a series of steps characterized by a single excited electron and the corresponding hole. The next step is that the excited elected electron and the hole are relaxed toward conduction band minimum and valence band maximum. By electron phonon interaction the electronic energy is lost to thermal energy in the nuclei by way of electron phonon interactions. This is what is happening in many bulk materials quite rapidly. In crystalline silicon this happens in pico seconds. In bulk materials the exciton binding energy is small. In nano- materials the exciton binding energy is large since the excited electron and hole are tightly bound. How the exciton can be dissociated into free charges in nano-materials is the important step to understand PV characteristics. These free charges could be transported independently in the opposite directions through the active layer to the metal contacts. Once the excited electron and hole are separated the next step is to understand how charge transport occurs. This may be through the temperature dependent hopping mechanism operating across the interfaces between nanomaterials (Hasobe et al., 2006; Lee et al., 2008; Hasobe et al., 2008).

\section{The electro-deposition route to produce thin film solar cells:}

In this section two kinds of deposition will be discussed. The first one is about Mist deposition. It is a method for depositing thin films using liquid precursors. It takes place at room temperature and atmospheric pressure. This technique allows precise control of the film thickness over larger areas than in the case of spin coating. This process also allows to pattern films during deposition which is not possible in the case of spin coating. In this process thinner films can be formed because of smaller droplet size. An electric field is applied to direct mist droplets the substrate as opposed to physical acceleration of solution out of the nozzle. Copper indium gallium (di) selenide is a I-III-VI compound semiconducting material composed of copper, indium, gallium and selenium (Altivisatos, 1996; Shanmugasundaram et al., 2008; Zhu et al., 2008). The material is a solid solution of copper indium selenide and copper gallium selenide, with a chemical formula of $\mathrm{CuIn}_{\mathrm{x}} \mathrm{Ga}_{1-\mathrm{x}} \mathrm{Se}_{2}$ where the value of $\mathrm{x}$ can vary from 1 (pure copper indium selenide) to 0 (pure copper gallium selenide). It is tetrahedrally bonded semiconductor with the chalcopyrite structure, band gap varying continuously with $\mathrm{x}$ from about $1.0 \mathrm{eV}$ (for copper indium selenide) to $1.7 \mathrm{eV}$ (for copper gallium selenide).

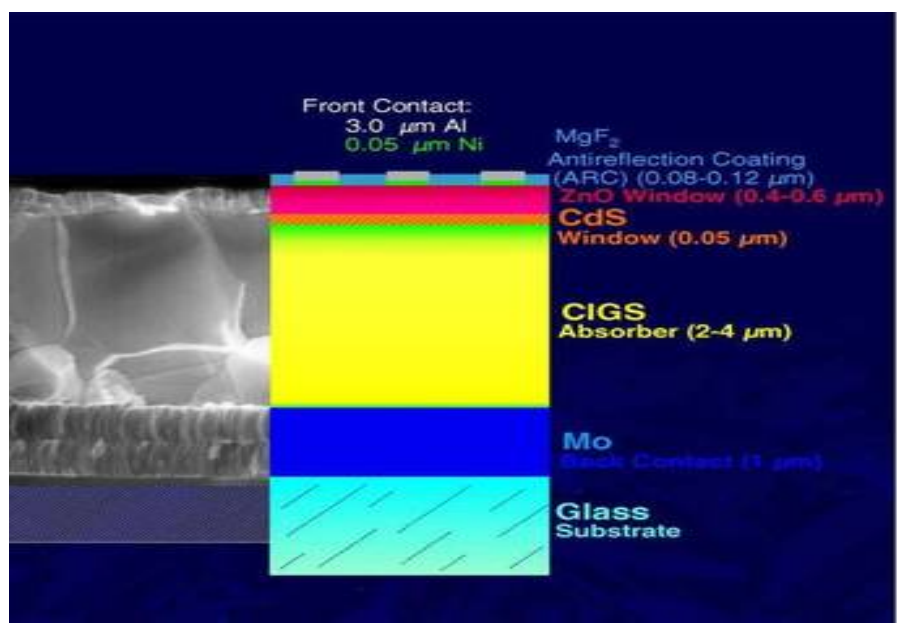

Figure 1: Structure of CIGS PV cell.

The basic structure of CIGS is depicted in the Figure 1. Molybdenum is coated over soda lime glass which is a substrate. Mo coating serves as metal back contact. The hetero-junction is formed between the semiconductors CIGS and ZnO buffered by a thin layer of CdS and a layer of intrinsic $\mathrm{ZnO}$. The CIGS is doped p-type from intrinsic defects, while the $\mathrm{ZnO}$ is doped n-type to a much larger extent through the incorporation of aluminum. This asymmetric doping causes the space charge layer to extend much farther into the CIGS than into the $\mathrm{ZnO}$. The wide CIGS layer serves absorber with a band gap between $1.02 \mathrm{eV}\left(\mathrm{CuInSe}_{2}\right)$ and $1.65 \mathrm{eV}\left(\mathrm{CuGaSe}_{2}\right)$.

Ion exchange methods are simple and attractive because of simplicity and low cost of the equipment and low temperature employed compared to vacuum deposition techniques. Copper has been incorporated into $\mathrm{CuInSe}_{2}$ by ion exchange process. The resulting solar cells using CIS do not yield high efficiencies. The work has been extended to incorporate $\mathrm{Cu}$ ions into indium gallium selenide (IGS) thin films and the properties of these films have been reported (Dloczik et al., 2003).

CIGS films can be manufactured by several different methods (Eberpacher et al., 2001; Banger et al., 2002). The most common method is vacuum based process. This is achieved by co-evaporation or co-sputtering of copper, gallium and indium. Annealing is done to the resulting film with a selenide vapor to form the final CIGS structure. Another method is to directly co-evaporate 
copper, gallium, indium, and selenium onto a heated substrate. Yet another process is a non-vaccum based process. This process is deposition of nano-particles of the precursor materials on the substrate and sinters them in situ. A company, Nano Solar uses copper indium gallium diselenide to build their thin film solar cells. The process involves a basic printing process with a semiconductor ink. The process does not utilize the expensive high vacuum based thin film deposition process. In Nano Solar's process, the ink is deposited on a flexible substrate (the paper) and the nano-component in the ink aligns themselves properly via molecular self assembly. This molecular assembly is a process by which molecules adopt a defined arrangement without guidance or management from an outside source. Molecular self assembly is an important aspect of bottom-up approach to nanotechnology. Using this approach the final (desired) structure is CIGS is a direct band gap material. Hence it has very strong light absorption and this material can be made use of in thin film (1-2 micro meters) solar cell unlike crystalline solar cells where greater thickness is required. The active layer (CIGS) can be deposited in a polycrystalline form. This process uses less energy than growing large crystals, which is a necessary step in the manufacture of crystalline silicon solar cells. Also these are, unlike crystalline silicon, flexible.

Recently a study, Kang et al. (2009), has been made wherein CIGS thin film absorbers are grown from Ga rich electrodeposited precursors, followed by selenization in Se vapor without an additional vacuum deposition process adjusting stoichiometry. It is possible to grow stoichiometric $\mathrm{CuIn}_{0.7} \mathrm{Ga}_{0.3} \mathrm{Se}_{2}$ thin film, with the expected band gap. The efficiency obtained for the PV device using the resulting compound CIGS absorber layer was only $2.5 \%$. PV nano-markets foresee a good trend and the growth will be most substantial. The next two years will be crucial in determining the market viability. Thin film will be most promising because the potential conversion efficiencies are just below that of crystalline silicon. The aim of this technology is to have manufacturing strategies that will offer a chance to reach sub dollar per watt manufacturing costs on both on glass and flexible modules. CIGS $_{2}$ is a chalcopyrite material with a near optimum band gap of $1.5 \mathrm{eV}$ and it has very high absorption coefficient. For a film of $2.7 \mu \mathrm{m}$ CIGS2 thin film prepared by sulfurization the efficiency obtained was $11.99 \%$. The effect of sodium addition on CIGS2 solar cells has been studied and the efficiency is reported to be increased (Vasekar and Dhere, 2009).

\section{Why pulse plating is necessary?}

Advances in power semiconductor technology manufacturing processes need pulse plating. This is essential because of precise design and for large scale integration which not only requires more flexibility and at the same time needs higher performance. The advantages of pulse plating as enumerated in (Chandrasekar and Malthy, 2008) are as follows: (1) Increase of the deposit homogeneity due to a higher throwing power even at complex shaped parts; (2) Dramatic improvement of the layer properties e.g the corrosion stability; (3) Acceleration of galvanic processes functionalizing by combination with dispersion coating; (4) By short cathodic pulses with a higher current density nucleation process can be increased; (5) By very short anodic pulses with a higher current density leveling and smoothening effect can be achieved; (6) Reduction of the deposited crystallized size starting from micron with a DC down to nano-crystallized size with pulse plating; (7) Controlling alloy composition just altering the pulse sequence.

What the pulse wave form does, will be seen below to understand the efficacy of the process:

Pulse wave form is an increase in the number of control variables over traditional direct current (DC). Direct current only helps to control the current density. But pulse current helps to control On time, Off time and Amplitude. More complex wave forms will provide us to control additional variables. These additional variables precisely provide us to control electrochemical process by affecting such properties as diffusion layer, grain size and nucleation. Usually in electro-plating a negatively charged layer is formed around the cathode. This layer charges to a known thickness. This prevents the ions from the bulk. In pulse electro deposition the output is periodically turned off. By this the layer is discharged. This helps easier passage of the ions through the layer and onto the cathode. Moreover, higher current density areas in the bath become more depleted than low current areas. During off time these ions migrate to the depleted areas in the bath. During on-time ions are evenly distributed and these are available to deposition. Pulse plating especially is useful in plating semiconductor materials. The grain size of CdSe films decreased at $6.25 \%$ duty cycle (Chandrasekar, 2008). In Pulse electro deposition of GaAs increase in duty cycle (10 to 50\%) produces smaller grain size and this helps in decreasing the band gap.

Cadmium free copper indium gallium sulfur selenide thin film solar cells have been reported using chemical bath deposited Zinc sulphide (Kundu, 2005). Shell Solar industries provided high quality CIGS absorber layers. The use of CBD-ZnS which is a higher band gap material than CdS improved the quantum efficiency of fabricated cells at lower wavelengths, leading to an increase in short circuit current. An attempt has been made to use the resulting complexes from the simplest possible selenoether of metal (Mishra et al., 2010). Me2Se, where the metal being copper, gallium and indium, is used as precursor for selenium containing chalcopyrite semiconductor material. An optimized general procedure for the high yield synthesis is described in Mishra (2010). CIGS thin films have been reported to be deposited by pulse laser deposition on flexible Cu foils (Jo et al., 2010). In this study a composite type microstructure of CIGS films of nanometer size in amorphous $\mathrm{Cu}$ rich matrix is reported to be achieved at high temperature of $500^{\circ} \mathrm{C}$. The scaling up process will be difficult in view of high temperature and cost. Moreover the average size of particle, shape, density and composition of CIGS film are strongly influenced by the substrate temperature.

The effects of gallium content in the CIGS absorber layer on the properties of the corresponding thin films have been studied (Jung et al., 2010). In this study solar cells were fabricated using a co-evaporation technique which is not helpful in scaling up 
process. The grain size of CIGS films decreased with increasing gallium content. This may be due to the difficulty of gallium diffusion during the second stage of the co evaporation process. In this process the short circuit current $J_{\mathrm{sc}}$ depends on $\mathrm{Ga} /(\mathrm{In}+\mathrm{Ga})$ ratio. Controlling this ratio will be very difficult in this process. To have optimum open circuit voltage $\mathrm{V}_{\mathrm{oc}}$ and at least $15 \%$ efficiency, optimum ratio has to be arrived at.

From the literature reports for semiconductor synthesis it is learnt that thin films nano crystalline photo electrodes with reasonable efficiencies can be easily deposited by the pulse plating technique in the presence of silico-tungestic acid. In this work it is stated that films with nano-crystallites in the range of 11-20 nm could be easily obtained. Among the several techniques for the deposition of CdSe films were successfully pulse electrodeposited with and without the use of hetero-polycyclic acid, viz., silico-tungstic acid. In these studies it is found that decrease of grain size was achieved. And grain size was also found to decrease when acid is added (Murali et al., 2009a). Zinc sulphide films could be pulse electro-deposited at a duty cycle $20 \%$ with different deposition current density $5 \mathrm{~nm}$ for the film is deposited (Murali et al., 2009b). Lastly the copper indium selenide films have been successfully electrodeposited onto indium tin oxide substrate and it is recently reported ( $\mathrm{Li}$ et al., 2010). The films are reported to be obtained at different pulse frequencies. The results also indicate that smooth surface and stoichiometric composition of the film is able to be attained at appropriate pulse frequency. Electro-deposition using pulse wave produces a faster deposition rate and also a better film adhesion ability than the traditional electro-deposition technique which will be a low cost thin film solar cell production.

\section{Conclusion}

In view of the demand for this film is going to be heavy, it is worthwhile to proceed appropriate manufacturing technology for CIGS films. As far as the present knowledge goes it is worthwhile trying to get these films by pulse electro-deposition. The cost of the resulting technology has to be looked into.

\section{References}

Altivisatos A.P. 1996. Perspectives on the physical chemistry of semiconductor nanocrystals, J.Phys. Chem, Vol. 100, p. 13226.

Banger K.K, Hollingsworth J.A, Harris J.D., Cowen. 2002. Ternary single-source precursors for polycrystalline thin-film solar cells, J. Appl. Organomet. Chem. Vol. 16, No. 11, p. 617

Chandrasekar M, Malthy P. 2008. Pulse and pulse reverse plating-Conceptual, advantages and applications, Electrochimacta, Vol. 53, p. 3313.

Su C.-Y., Ho W.-H., Lin H.-C., Nieh C.-Y., and Liang S.-C. 2011. The effects of the morphology on the CIGS thin films prepared by CuInGa single precursor, Solar Energy Materials and Solar Cells, Vol. 95, No. 1, pp. 261-263.

Dloczik. L, Lux-Steiner M. C., and Koenenkamp R. 2003. Study on the preparation of structured $\mathrm{CuInS}_{2}$ films by ion exchange processes, Thin Solid Films, Vol. 431-432, pp. 131-134.

Eberpacher C, Fredric C, Pauls K, Serra J. 2001. Thin-film CIS alloy PV materials fabricated using non-vacuum, particles-based techniques, Thin Solid Films, Vol. 387, p. 18.

Kang F., Ao J., Sun G., He Q., and Sun Y. 2010. Properties of CuInxGa1-xSe2 thin films grown from electrodeposited precursors with different levels of selenium content, Current Applied Physics, Vol. 10, No. 3, pp. 886-888.

Gur I. Fromer N.A., Geier M.L., Ailvisatos A.P. 2005. Air-stable all-inorganic nanocrystal solar cells processed from solution, Science, Vol. 310, No. 5747, p. 462.

Hasobe T. 2010. Supramolecular nanoarchitectures for light energy conversion, Phys.Chem. Vol. 12, p. 44.

Hasobe T., Fukuzmi S., Kamat P.V., 2006. Hierarchical assembly of porphyrins and fullerenes for solar cells, Interface Vol. 15, p. 47.

Hasobe T., Oki A., Sandanayaka S.D., Murata H. 2008. Sonication-assisted supramolecular nanorods of meso-diaryl-substituted porphyrins, Chem. Commun., p. 724.

Hermann A.M., Gonzalez C., Ramakrishnan P.A., Balzar D., Popa N., Rice P., Marshall C.H., Hilfiker J.N., Tiwald T., Sebastian P.J., Calixto M.E., and Bhattacharya R.N. 2001. Fundamental studies on large area $\mathrm{Cu}(\mathrm{In}, \mathrm{Ga}) \mathrm{Se}_{2}$ films for high efficiency solar cells, Solar Energy Materials and Solar Cells, Vol. 70, No. 3, pp. 345-361.

Kamat P.V., 2008. Quantum dot solar cells. semiconductor nanocrystals as light harvesters, J.Phys. Chem., Vol. 112, p. 18737.

Kanai, Y., Wu, Z., Grossman J.C., 2010. Charge separation in nano-scale photovoltaic materials: recent insights from first principles electronic structure theory, J. Mater.Chem., Vol. 20, p. 1053.

Kang, F., Ao J.P., Sun, G.Z., He Q., Sun Y., 2009. Preparation and properties of CuInxGa1-xSe 2 thin-film solar cell absorbers from selenization of Ga-rich electrodeposited precursors, Semicond.Sci.Technol. Vol. 24, No. 1.

Kavcar, N., Carter M.J., Hill R. 1992. Characterization of $\mathrm{CuInSe}_{2}$ thin films produced by thermal annealing of stacked elemental layers, Solar Energy Materials and Solar Cells, Vol. 2, p. 13.

Lee S.J., Hupp J.T., Nguyen S.T. 2008. Growth of narrowly dispersed porphyrin nanowires and their hierarchical assembly into macroscopic columns, J.Am.Chem.Soc., Vol. 130, p. 9632. 
Li W.X, Guangjun W, Bao-Li, T, Shaoming W, Du Z. 2010. CuInSe ${ }_{2}$ thin films obtained by pulse-plating electrodeposition technique with novel pulse wave, Chinese Science Bulletin, Vol. 55, No. 18, p. 1854.

Murali K.R., Manoharana C., Danapandiana S., 2009a. Photoelectrochemical properties of pulse electrodeposited cadmium selenide films, Chalcogenide Letters, Vol. 6, No. 1, p. 57

Murali K.R, Manoharana C, Danapandiana S. 2009b. Pulse electrodeposited zinc selenide films and their characteristics, Chalcogenide Letters, Vol. 6, No. 1, p. 51

Dhere N.G. 2011. Scale-up issues of CIGS thin film PV modules, Solar Energy Materials and Solar Cells, Vol. 95, No. 1, pp. 277 280.

Panthani, M.G; Akhavan, V; Goodfellow ,B; Schmidke, J.P; Dunn, L; Dodabalapur, A; Barbara, P.F; Korgel, B.A. (2008): Synthesis of $\mathrm{CuInS}_{2}, \mathrm{CuInSe}_{2}$, and $\mathrm{Cu}(\mathrm{InxGa1}-\mathrm{x}) \mathrm{Se}_{2}$ (CIGS) Nanocrystal "Inks" for Printable Photovoltaics. J. Am. Chem. Soc., Vol. 130, No. 49, p. 16770.

Vasekar P.S., and Dhere N.G., 2009. Effect of sodium addition on Cu-deficient CuIn1-xGaxS2 thin film solar cells, Solar Energy Materials and Solar Cells, Vol. 93, No. 1, pp. 69-73.

Powalla M., and Dimmler B. 2001. CIGS solar cells on the way to mass production: Process statistics of a $30 \mathrm{~cm} \times 30 \mathrm{~cm}$ module line, Solar Energy Materials and Solar Cells, Vol. 67, No. 1-4, pp. 337-344.

Kundu S., and Olsen L.C. 2005. Chemical bath deposited zinc sulfide buffer layers for copper indium gallium sulfur-selenide solar cells and device analysis, Thin Solid Films, Vol. 471, No. 1-2, 3, pp. 298-303.

Ahn S., Kim K., and Yoon K.H., 2008. Nanoparticle derived $\mathrm{Cu}(\mathrm{In}, \mathrm{Ga}) \mathrm{Se}_{2}$ absorber layer for thin film solar cells, Colloids and Surfaces A: Physicochemical and Engineering Aspects, Vol. 313-314, pp. 171-174.

Shanmugasundaram, K; Price,S.C; Li,W; Jiang ,H; Huang, J; Wang, Q.K; Yang,Y; Ruzyllo, J. 2008. Studies of mist deposition in the fabrication of blue organic light emitting diodes, Semicond.Sci .Technol., Vol. 23, 075036

Mishra S., Jeanneau E., and Daniele S. 2010. Dimethyl selenide complexes of copper, gallium and indium halides a potential precursors for selenium-containing chalcopyrite semiconducting materials, Polyhedron, Volume 29, Issue 1, 13 January 2010, pp. 500-506.

Ishizuka S., Yamada A., Matsubara K., Fons P., Sakurai K., and Niki S. 2010. Development of high-efficiency flexible $\mathrm{Cu}(\mathrm{In}, \mathrm{Ga}) \mathrm{Se} 2$ solar cells: A study of alkali doping effects on CIS, CIGS, and CGS using alkali-silicate glass thin layers, Current Applied Physics, Vol. 10, No. 2, Supp. 1, March, pp. S154-S156.

Jung S., Ahn S.J., Yun J.H., Gwak J., Kim D., \& Yoon K.. 2010. Effects of Ga contents on properties of CIGS thin films and solar cells fabricated by co-evaporation technique, Current Applied Physics, Vol. 10, pp. 990-996.

Wang, Y; Herron, N., 1991. Nanometer-sized semiconductor clusters: materials synthesis, quantum size effects, and photophysical properties J. Phys. Chem. Vol. 95, p. 525.

Wu Y., Wadia,C., Ma W.L, Sadtler B., Ailvisatos A.P. 2008. Synthesis and photovoltaic application of copper (I) sulfide nanocrystals, Nanoletters, Vol. 8, No. 8, p. 2551.

Jo Y.H., Mohanty B.C., \& Cho Y.S. 2010. Crystallization and surface segregation in CuIn0.7Ga0.3Se2 thin films on $\mathrm{Cu}$ foils grown by pulsed laser deposition, Applied Surface Science, Vol. 256, No. 22, pp. 6819-6823.

Oda Y., Matsubayashi M., Minemoto T., and Takakura H., 2010. Fabrication of $\mathrm{Cu}(\mathrm{In}, \mathrm{Ga}) \mathrm{Se}_{2}$ thin film solar cell absorbers from electrodeposited bilayers, Current Applied Physics, Vol. 10, No. 2, Supp. 1, pp. S146-S149

Zhu, T; Shanmugasundaram, K, Price S.C, Ruzyllo J, Zhang F, Xu, J., Moheney S.E, Zjang Q, Wang A.Y., 2008. Mist fabrication of light emitting diodes with colloidal nanocrystal quantum dots, Appl. Phys. Lett. 92, 023111.

\section{Biographical notes}

Dr. N.G. Renganathan was a Senior most Scientist from Central Electro chemical research Institute, Karaikudi. He has post graduation from I.I.T. Bombay. He got his Ph.D. from Madurai Kamaraj University. After superannuation he is now working as Professor in Vel Tech. University, Avaid, Chennai. He has more than 60 papers to his credit.

Mr. M. Venkatasubramanian is working as Assistant Professor in Vel Tech University, Avadi, Chennai. He has post graduation from I.I.T. Bombay. He was R\&D Manager in Chempon dyes.

Dr..S..Mohan is a Senior Scientist from Central Electrochemical Research Institute, Karaikuidi. He has post graduation from Madras University. He got his Ph.D. in physical chemistry from I.I.T. Madras. He has more than 20 years experience at C.E.C.R.I, Karaikudi and he has more than 80 papers to his credit and 3 patents.

Received June 2010

Accepted May 2011

Final acceptance in revised form May 2011 